\section{MS38-P12 Approaching the structure of tetranuclear Ni-complex: a journey through the maze of non-crystallographic information}

Dubravka Sisak Jung ${ }^{1}$, Ana Belen Pinar Prieto ${ }^{2}$, Predrag Lazic ${ }^{3}$, Gordana Pavlovic ${ }^{4}$, Marina Cindric ${ }^{5}$

\author{
1. DECTRIS Ltd, Baden, Switzerland \\ 2. ETH Zurich, Switzerland \\ 3. Institute Rudjer Boskovic, Zagreb, Croatia \\ 4. Faculty of Textile Technology \\ 5. Faculty of Natural Sciences and Mathematics, Zagreb, Croatia
}

email: dubravka.sisak@dectris.com

As a result of various synthetic paths, a cubane-type $\mathrm{Ni}$-complex was obtained. Its single-crystal structural analysis revealed four nickel atoms coordinated with five donors from organic ligand and one methanol molecule. Heating this sample to $180^{\circ} \mathrm{C}$ lead to a change of magnetic properties from ferro- to antiferromagnetic, and of color from green to red. For clarity, the former sample will be referred to as cubane-Ni, and the latter as cubane-Ni-180. In order to solve the structure of cubane-Ni-180, X-Ray Powder Diffraction (XRPD) data were measured using the MYTHEN detector installed at Swiss Light Source. Any radiation damage was avoided by collecting $120^{\circ}$ of data within ten seconds. However, the data resolution was only $3 \AA$. This, combined with lack of molecular model hindered classical structure determination, so additional information had to be considered. Thermogravimetric analysis showed a single weight loss, indicating that all methanol molecules were removed from the cubane-Ni structure upon heating. In fact, when the cubane-Ni-180 was exposed to a methanol atmosphere, it transformed back to cubane-Ni. These observations, supported by IR spectroscopy results, suggested that the Ni-ligand moiety was preserved. The XRPD patterns of both samples were found to be similar, so the unit cell parameters of the cubane-Ni could be used to facilitate the indexing of the cubane-Ni-180 pattern, which exhibited very few peaks. Quantum mechanical methods were subsequently used to assist in the structure solution. First, a starting structural model was built by removing the methanol ligands from the cubane-Ni structure. This model was subjected to series of energy optimizations, all resulting in clusters of four square-planar coordinated Ni-centers. This geometry was found to comply with the antiferromagnetic properties of the sample. Notwithstanding that the molecular structure was reasonable, the fit between the calculated and measured XRPD patterns was not satisfactory. In order to exclude the possibility of a strong model-bias, direct-space optimization of the modified molecular structure of cubane-Ni was tried. The last obstacle, preferred orientation of the crystallites, was addressed by adding geometrical restraints to the optimization. This way, solutions with chemically reasonable intermolecular distances could be favored. Finally, examination of the structures obtained by both approaches allowed the most likely structure of cubane-Ni-180 to be proposed.

Keywords: X-ray Powder Diffraction, structure determination, TG, quantum mechanics, Nickel complex, combining methods

\section{MS38-P13 On element sensitive determination of local atomic structure with white beam X-ray fluorescence holography \\ Dawid T. Dul ${ }^{1}$, Paweł Korecki ${ }^{1}$}

1. Institute of Physics, Jagiellonian University, Łojasiewicza 11, 30-348 Kraków, Poland

email: dawid.dul@gmail.com

White beam x-ray fluorescence holography (white beam $\mathrm{XFH}$ ) enables local atomic structure determination around particularly chosen elements in the sample. It combines $\mathrm{x}$-ray diffraction and $\mathrm{x}$-ray fluorescence spectroscopy to obtain a hologram of the crystal structure around the element of interest. The hologram is formed as a result of the interference of the incident X-ray wave with waves scattered from atoms inside the sample. It is probed at the positions of absorbing atoms which work as internal detectors by measuring $\mathrm{x}$-ray fluorescence photons. Structure information can be obtained directly from the holograms, since due to the short coherence length of white $\mathrm{x}$-rays they can be interpreted as quasi-real space projections of the crystal structure, or through the application of reconstruction procedures [1, 2].

In this work, by employing analytic and numerical calculations, we show that element sensitive determination of local atomic structure with white beam $\mathrm{XFH}$ is generally only possible if so called matrix effects i.e. beam attenuation and indirect excitation are properly tackled [3]. We argue that these effects may lead to distortions in the holographic signal and/or spurious maxima in the reconstruction. Furthermore, we demonstrate than the effect of beam attenuation on the holograms is very weak for high incident beam energies whereas indirect excitation is important regardless of the energy range. We use these finding and propose an approximate, yet efficient method of correction for matrix effects [4].

This work was supported by the Polish National Science Center (DEC-2013/09/N/ST3/04111) and in part by the KNOW Research Consortium through the Marian Smoluchowski fellowship.

[1] P. Korecki, et al., Physical Review Letters 96, 035502 (2006)

[2] P. Korecki, et al., Journal of Synchrotron Radiation 18, 851 (2011).

[3] D. T. Dul, et al., EPL (Europhysics Letters) 104, $66001(2013)$

[4] D. T. Dul, et al., Journal of Applied Crystallography, in press (2015).

Keywords: x-ray diffraction, x-ray fluorescence, local atomic structure 